\title{
County-level Recruitment in Online Samples: Applications to COVID-19 and Beyond
}

\author{
Leib Litman ${ }^{1,3}$, Robert O. Hartman ${ }^{4}$, Shalom N. Jaffe ${ }^{1,3}$, and Jonathan Robinson ${ }^{2,3}$ \\ ${ }^{1}$ Department of Psychology, Lander College \\ ${ }^{2}$ Department of Computer Science, Lander College \\ ${ }^{3}$ CloudResearch \\ ${ }^{4}$ The MITRE Corporation
}

\section{Author Note:}

Leib Litman: (iD https://orcid.org/0000-0001-6598-2701

Robert O. Hartman: (D) https://orcid.org/0000-0001-8499-7570

Shalom N. Jaffe: (iD https://orcid.org/0000-0002-7523-5213

Jonathan Robinson: (iD https://orcid.org/0000-0001-5353-7969

Correspondence concerning this article should be addressed to Leib Litman, Department of Psychology, Lander College, 75-31 150 ${ }^{\text {th }}$ St., Kew Gardens Hills, NY, United States. Email: leib.litman@touro.edu 


\begin{abstract}
Thousands of readily downloadable county-level data sets offer untapped potential for linking geo-social influences to individual-level human behavior. In this study we describe a methodology for county-level sampling of online participants, allowing us to link the selfreported behavior of $\mathrm{N}=1084$ online respondents to contemporaneous county-level data on COVID-19 infection rate density. Using this approach, we show that infection rate density predicts person-level self-reported face mask wearing beyond multiple other demographic and attitudinal covariates. Using the present effort as a demonstration project, we describe the underlying sampling methodology and discuss the wider range of potential applications. Keywords: county-level data, online sampling, COVID-19
\end{abstract}




\section{County-level Recruitment in Online Samples: Applications to COVID-19 and Beyond}

Social-behavioral research has traditionally been conducted using predominantly university subject pools and community samples, a practice widely criticized as lacking diversity (e.g. Gosling \& Johnson, 2010). Less often discussed is the lack of flexibility with respect to targeting specific proportions of demographic groups or geolocations. Despite an intensive, decades-old debate about the representativeness (or lack thereof) of university subject pools (e.g. McGuire, 1967; Oakes, 1972; Ashton \& Kramer, 1980; Sears, 1986; Abelman, 1996; see also Moss et al., 2020), issues of sample composition were largely ignored in psychology and other behavioral sciences for over 50 years. During that time, sample composition was typically left almost entirely up to chance (i.e., the characteristics of the university student population), with researchers employing an implicit 'first-through-the-door' approach to sample composition. However, as so-called "WEIRD" participants came under greater scrutiny (e.g. Henrich et al., 2010), issues of sample composition have begun to receive broader attention.

Starting in the 1990 s, online recruitment began to provide access to a wider and more diverse participant pool by allowing researchers to quickly and inexpensively access participants from across the United State and even the world (Smith \& Leigh, 1997; Nosek et al., 2002; see Gosling, et al., 2004). But it wasn’t until around 2010 that online recruitment gained wide adoption, owing primarily to the rise of Mechanical Turk and other services (Buhrmester et al., 2011). As an example of the widespread adoption of online sampling by researchers, since 2010 , studies that used Mechanical Turk for participant recruitment have now been published in over 1,000 different peer-reviewed journals (Buhrmester et al., 2018).

Starting in 2016, Mechanical Turk made it possible to directly recruit individuals based on their demographics and other characteristics. This made it easier to target and oversample for 
specific participant demographic categories, including hard-to-sample ethnic/cultural groups, specific ages, individuals with military backgrounds, shopper profiles, and many other characteristics. Nevertheless, while these tools have substantially increased researchers' options for achieving demographic diversity, capabilities for achieving geographic diversity are more limited.

Geographic diversity of samples can be important in at least three ways. First, certain research questions require control over geographic regions such as cities or states from which participants are sampled. For example, Plaut et al. (2012) examined how self-evaluation is differentially associated with education and social status in Boston and San Francisco, two cities that were specifically targeted because of their contrasting cultural backgrounds. Second, geographic diversity can be important for generalizability. For example, people on the East and West coasts of the United States have differing attitudes toward weather, terrain, and climate change (Plaut et al., 2012). Because such phenomena covary with region, having control of the geographic locations from which participants are sampled can help to reduce bias. Finally, geographic location can be an important predictor of political, health, and economic outcomes, and can thus be a critical aid to substantive hypothesis-testing. In this study, we describe a repeatable, customizable methodology for achieving fine-grained geographic diversity at the county level in online samples, with the recent COVID-19 outbreak serving as our focal test case.

\section{The Present Study}

It has been widely reported that people differ with regard to their willingness to wear face masks during the COVID-19 pandemic (e.g. Frankovic, 2020). In particular, differences in face mask wearing, as well as other attitudinal and behavioral differences toward COVID-19, have 
been attributed to such socio-cultural factors as political partisanship and education (Frankovic, 2020). We postulated that geographical factors, such as regional population density in general or COVID-19 infection rate density (defined as the number of positive cases reported at a specific time divided by the region's population) in particular, might add explanatory power to these person-level factors. To test this hypothesis, however, we needed some level of control over the regions from which participants were sampled.

Here we describe a methodology for obtaining a geographically diverse sample based on county-specific COVID-19 infection density rates. Specifically, we defined 'hot' and 'cold' regions of COVID-19 infection density at the local county level and controlled how participants are sampled from these zones. Infection density rates vary widely across different regions of the country. Thus, collecting a geographically diverse sample in which participants were recruited equally across different infection density rate zones allowed us to examine whether demographic, political, attitudinal, and regional predictors make independent contributions to COVID-19 health-related outcomes.

\section{County-level Data}

The United States is divided into approximately 3143 counties and county-equivalents, which act as the primary political and administrative divisions of States. Various government agencies routinely collect data at the county level, most of which are freely available from government websites and data repositories (see Jia et al., 2004; Killeen et al., 2020). The extensive county-level data collected by the US Census is perhaps the most prominent example, but it is far from alone. For example, the US Department of Agriculture maintains county-level data on poverty rates, income, unemployment, and education, and the Agency for Healthcare Research and Quality provides numerous health statistics, also available at the county level. 
Finally, during the COVID-19 pandemic, the Center for Disease Control (CDC) has regularly collected infection and death rates across the United States, which it makes available at the county level. For the present study, we leverage this last data source to define regions of high, medium, and low infection density rates, which we use to inform our online data collection sampling strategy.

\section{General Methodological Approach}

Using county-level COVID-19 infection rate data, we divided the United States into high, medium, and low infection density zones. The high infection density rate zone was defined as the top $10 \%$ of counties with the highest infection density rates, accounting for 82 million US residents. The medium zone consisted of counties with infection density rates in the 90th to 20th percentiles, representing 250 million residents. The low zone comprised counties in the bottom $20 \%$ of county-level infection density rates and included 20 million residents. These thresholds were chosen so that a meaningfully large proportion of the population would be included in each group. Specifically, we sought to vary the high and low infection density zones to represent a minimum of $10 \%$ of the counties and $5 \%$ of the US population.

Having defined these three zones, we launched an online study that sampled a third of its participants from each zone. We hypothesized that county-level infection density would exhibit a positive relationship with individual-level face mask wearing. Specifically, people who live in counties with high infection density rates at the time of the study would be more likely to wear masks compared to those who live in counties with lower infection density rates. We additionally predicted that county-level infection density would remain a significant predictor of mask wearing over and above widely discussed individual-level demographic and attitudinal covariates, such as political orientation, party affiliation, and educational background. Finally, 
although we had no strong hypothesis, we wished to determine whether county-level infection density would remain a significant predictor even after accounting for county-level population density. That is, we anticipated the possible objection that COVID-19 infection density was acting as a mere proxy for generic population density, and we wanted to examine this issue.

\section{Method}

\section{Participants and Procedure}

Data were collected between May 20th and May 25th, 2020. We recruited 1018 participants from the three aforementioned United States regions with different COVID-19 infection densities (high infection density, $\mathrm{N}=345$; medium, $\mathrm{N}=353$; low, $\mathrm{N}=325$ ). All participants completed a survey (programmed in Qualtrics) containing demographic questions and several measures of self-reported attitudes and COVID-19-focused behaviors. The study took 25 minutes to complete on average. The order of all stimulus blocks was randomized, except for the demographic block, which was presented last for all respondents. Sample demographics for gender, age, race, ethnicity, education, political party affiliation, and political orientation are presented in Table 1.

\section{Outcome Measures}

The outcome measure was a binary yes/no question asking respondents whether they had started to wear a protective face mask in direct response to the COVID-19 pandemic.

Respondents saw the following introduction: "Comparing your behavior in recent weeks to your typical behavior before you heard about the coronavirus (COVID-19), how much have you recently increased or decreased each of the following behaviors?" The focal question was "Have you worn a medical/surgical style of face mask in public?"

\section{Additional Measures not Reported in This Study}


Multiple other measures were used in this study, including measures of actively openminded thinking, need for cognition, the "big five" personality factors, conspiracist ideational tendencies, and perceptions about the credibility of science. These measures, which were collected as part of a study examining the relationship between attitudes toward science and COVID-19 behavior, are the subject of a separate report in preparation.

\section{Data Source}

Participants were recruited from Prime Panels (e.g. Ballew et al., 2019; Job et al., 2018; Davidai, 2018; Waggoner, 2018, Deri, et al., 2017; Chandler et al., 2019), an online participant recruitment platform. Prime Panels accesses participants via automated API integration with hundreds of other online data providers, making it possible to reach more potential respondents in various parts of the country. Prime Panels also leverages several sophisticated data quality screening capabilities, which are critical to collecting accurate self-report data from online panels (see Chandler, et al, 2019 for a detailed discussion). Respondents can be recruited on Prime Panels via multiple targeting variables, including designated market area (DMA; see below) and zip code.

\section{County-level Targeting}

Online participant platforms vary in the mechanism that enables geographically granular recruitment. Generally, there are three targeting strategies to facilitate county-stratified sampling: recruitment directly by county, by zip code, or by Designated Market Area (DMA). DMAs are regions created by Nielsen that correspond to multiple counties and zip codes that can receive the same television, radio, and broadcast channels (Goldsbery, 2017). Because of their extensive use in advertising and marketing research, market research panel platforms typically are more oriented toward DMA-based targeting than literal county or zip code targeting. In contrast, 
health and economic data typically are collected by the federal government at the county level, where the concept of DMA has little if any currency. The upshot of this is that the use of market research-centric panels for county-level targeting usually requires an intermediate step of translating counties to corresponding DMAs or zip codes.

For our use case, we matched county-level COVID-19 infection density data to zip codes. Both DMAs and zip codes often span multiple counties, complicating the matching process. Most commonly, zip code areas are smaller than counties and are contained within a single county. Thus, in most cases, all zip codes within a specific county were assigned the COVID-19 infection rate density level of that county. However, there are times when a specific zip code spans multiple counties. In these cases, the COVID-19 infection rate density levels of the multiple counties which corresponded to a specific zip code were averaged.

County-level COVID-19 data was obtained from usafacts.org, which provides weekly infection rates updates available for download as CSV files. The infection rates in each county were adjusted to each county's population by a simple conversion to percentages based on each county's population size (county population size is also available from usadata.org). Those county-level parameters were then merged with a list of $\approx 42,000$ zip codes spanning the entire United States. The zip code data was sorted by infection rate, with the top $10 \%$ of the highest infection rate zip codes constituting the high-infection rate density group and the lowest $20 \%$ of zip codes with the lowest rates defining the low-infection density rate group. Finally, the zip codes in the middle $70 \%$ of infection rates were deemed the medium-infection density group. All $\approx 42,000$ zip codes were then uploaded to Prime Panels and quotas were placed on the three groups such that no more than a third of the sample could come from any one of them. Thus, when Prime Panels detected that 333 participants came from any of the zip codes in group 
1, data collection was automatically stopped for that region. Prime Panels automatically collected data on which zip code a respondent was taking the survey from. These zip codes, and their infection density rates, were merged with the survey data file for analysis. The infection density rate of each participant's zip code was used as the main predictor in all regression models.

\section{Analytic Approach}

Each participant's zip code infection rate was added to the survey data file, allowing infection density to be a subject-level variable in regression models. After reviewing descriptive statistics, logistic regressions were run in three model sets to examine whether infection density was a predictor of face mask wearing. For Model Set 1 regression models were run separately for the following predictors: gender, age, race (White being the reference category), Hispanic ethnicity, education (No college degree being the reference category), income, political party affiliation (Republican being the reference category), political affiliation, county-level infection density, and county population. The Model Set 2 regression included all of the above-mentioned predictors as covariates, but not the county level covariates (infection rate density and county population). In Model Set 3, county-level infection density and county population were entered as predictors in addition to the demographic predictors from step 2 (age, gender, political affiliation, race, ethnicity, income, and education). Logistic regression was used due to the binary outcome measure.

\section{Results}

\section{Descriptive Statistics for the Three COVID-19 Density Regions}

Table 1 shows the differences in face mask wearing, political party affiliation, political orientation, and region across the three COVID-19 density zones. People from the high-density 
COVID-19 infection density rate zone (high-density) were 14 percentage points more likely to wear masks compared to people from low-density zones (see Table 1).

Perhaps the most notable difference across the zones was in settlement type of residence (see Figures 1 and 2). Seventy-five percent of people in high-density regions reported living in at least medium-size cities with over 50,000 residents or in suburbs surrounding large cities (see Figure 1). This was completely reversed for the low-density regions, in which $80 \%$ of respondents reported living in small towns of fifty-thousand people or fewer, either on a farm or in open country areas.

Figure 2 shows average county-level infection density rate by community type. Large cities with over 250,000 residents contained the counties with the highest infection density rate densities on average $(M=1.08, S D=.80)$, followed by suburbs near a large city $(M=0.83, S D$ $=.81)$, medium size cities $(\mathrm{M}=0.55, \mathrm{SD}=.71)$, small cities with fewer than 50,000 residents $(\mathrm{M}$ $=0.33, \mathrm{SD}=.59)$, farms $(\mathrm{M}=0.19, \mathrm{SD}=.34)$ and open country areas $(\mathrm{M}=0.20, \mathrm{SD}=.39)$. All areas were significantly different $(\mathrm{p}<0.05)$ from each other with the exception of open country areas and farms.

The political (see Table 1) and demographic (see Table 2) composition of people living in these three density zones also differed in substantial and predictable ways. People in high-density regions were more likely to be Democrats, to have a liberal orientation, to have higher household incomes, and to have higher education levels. These regions also were more ethnically diverse, with more African-American and Hispanic respondents.

\section{County-level Infection Density Rates as a Predictor of Face Mask Wearing}

Results from Model Set 1 (each predictor examined separately without any covariates) showed that several factors were predictive of self-reported face mask wearing. These were 
county-level infection rate density, county-level population, political party affiliation (Republican vs. Democrat), political orientation (on a liberal/conservative scale), income, education, and age (see Table 3 coefficients and Odds Ratios). For Model Set 2, in which all except for county-level predictors were entered as covariates in the model, political party affiliation (Democrat vs. Republican) and age remained significant. Finally, Model Set 3 added the county-level predictors to all predictors from Set 2, and only age and county-level infection rate density remained significant in the presence of the other covariates (see Table 3 coefficients and Odds Ratios).

\section{Discussion}

In this study we defined three US zones based on COVID-19 infection rate density, using county-level data from the Centers for Disease Control. Having defined these zones, we collected an online sample in which the number of respondents from these zones was controlled. This approach ensured that the sample contains an equal distribution of participants living in high, medium, and low COVID-19 infection density zones.

The infection rate density zones had specific demographic, regional, and political profiles. The high infection rate density zone was primarily from high-population urban regions. Sixty percent of respondents from the high-density zone lived in either large cities with more than 250,000 residents or in surrounding regions. In comparison, fewer than $10 \%$ of participants from the low-density regions lived in these areas. This pattern corresponds to what is known about COVID-19 hotspot outbreak zones being much more likely to have occurred in large cities at the time the data for this study was collected. The demographic and political profile of different infection rate density zones also corresponded to the types of differences typically found between large cities and rural areas. High infection rate density zones tended to be more 
left-leaning politically and more likely to identify with the Democratic party, to have higher levels of education, to have higher incomes, and to have more minorities.

Using county-level COVID-19 infection density rates as predictors of mask wearing, we found that the infection rate density in the counties where respondents took the survey uniquely predicted self-reported mask-wearing. Indeed, of the multiple variables that predicted face mask wearing, only age and county-level infection density rates remained significant in the presence of other covariates. For example, when analyzed in models that excluded other predictors, Democrats, bachelor's degree holders, and higher-income respondents all were significantly more likely to wear a mask than their counterparts—Republicans, those with no degree, and lower-income respondents, respectively. However, those differences disappeared in the presence of county-level covariates. This suggests that person-level demographic, political, and achievement variables may be acting largely as proxies for broader cultural/geographical differences.

This makes some sense: Republicans, those without college degrees, and lower-income individuals all are more likely to live in rural areas, where infection rate density was relatively low at the time of data collection. Our data show that, in such regions, people from all groups, including Republicans and Democrats, are significantly less likely to wear face masks than those living in high-density regions. This implies that models that do not directly control for regional infection rate density may suffer from omitted variable bias. Importantly, having included the county population sizes in the model, we were able to show that it is not regional population density alone, but specifically the density of infection rate within a region, that uniquely predicts the likelihood of wearing a mask. 
Importantly, we are not suggesting that these results provide a definitive demonstration that there are no "real" differences in face mask wearing nationwide between these demographic groups. Our aim with this study is not to formulate and test a complex path model of the relationship among these variables. What our results do show, more modestly, is that regional infection rate density is an important covariate to consider in such hypothesis-testing and modelbuilding efforts whenever a behavior covaries not only with demographic variables but also with other geographically varying phenomena (in this case, infection rate density). In summary, the present study serves as a test case for a methodology that allows for geographically-oriented modeling in online data collection via county-level sampling through zip codes. In particular, the present results demonstrate the feasibility of county-level targeting in the United States in online studies and sampling from large US regions that are defined by county-level parameters. The results also illustrate how county-level statistics can be used as covariates (i.e., control variables) in statistical models of attitudes and behavior collected in online surveys, which can improve the accuracy of statistical estimates for the substantive predictors of interest.

\section{Applications of County-level Sampling in Online Studies}

County-level data collection methods like those described in this study are rarely, if ever, utilized in online opt-in panel research, despite the potential applicability of this methodology to numerous research domains across the social and behavioral sciences. County-level datasets are available for a very large number of variables that can be of interest to social, behavioral, and health scientists (e.g. Jia et al., 2004; Hu \& Rao, 2009; Eichstaedt et al., Beaver \& Wright, 2011; Lawless \& Lucas, 2011; 2015; Killeen et al., 2019). However, county-level data are typically used by merging multiple existing datasets rather than for the collection of new data. For example, Kim et al., (2006) constructed a measure of social capital based on data available at the 
county level in existing datasets and showed that social capital is associated with obesity and physical activity. Muramatsu (2003) showed that poverty rates at the county level predict depression rates. Other county-level studies examined psychological distress (Dhingra et al., 2009), health outcomes (Rettenmaier \& Wang, 2013), mental health (Ells et al., 2009), and racial bias (Riddle \& Sinclair, 2019).

These studies illustrate how data already captured in national datasets can be used in behavioral research. However, while there are numerous extant datasets available, those datasets more often than not do not have the information that researchers may need to answer a specific research question. For example, using county-level data on social capital provided by Muramatsu et al, researchers can study the relationships between social capital and variables that cannot be accessed in extant datasets. Thus, expanding the geographic diversity of online sampling would allow researchers to harness the vast array of data available in national datasets, and to expand that research to questions pertaining to variables not currently available in those data sets.

\section{Limitations and Future Directions of County-level Sampling}

Several limitations of county-level data collection should be pointed out. First, counties differ significantly with regard to their size. While some counties have as few as a thousand residents, others have millions of residents. For example, Los Angeles County, the largest in the United States, has more than ten million residents, and each borough of New York City is also its own county. For counties as large as Los Angeles or Brooklyn, average estimates of poverty, health, and outcomes such as infection rates examined in this study can be misleading. Such large counties have multiple neighborhoods that can significantly differ from each other across multiple outcomes that are merged together at the county level. Another limitation of county- 
level datasets is that significant effort is often required to make sure that national datasets contain accurate records (e.g. Pridemore, 2005).

Several county-level predictors that were not examined in the current study should be explored in future research. While the present study focuses on infection rate density, it may also be that death rate density plays an independent role as a predictor of health behaviors. COVID19 death rates vary by region and are influenced by socio-economic and other county-specific factors. Thus, future studies should examine whether county-level COVID-19 death rates are an independent predictor of health behavior above and beyond infection rate density.

The historical trajectory of infection rate density within specific regions may be another important factor to explore in future studies. There is considerable variability in infection rate density over time within any specific region. For example, Northeast regions such as New York and New Jersey had very high infection density rates during the start of the pandemic, but then those rates decreased as other historically low infection rate density regions increased over time. While our current data do not speak directly to the underlying cause that drives the relationship between infection rate density and health behaviors, we hypothesize that the perception of present danger and a sense of vulnerability is what makes people in high infection density zones adhere more strictly to recommended health practices. To the extent that the perception of present danger and a sense of vulnerability plays a causal role in influencing health practices, people living in regions that have had historically low infection rates may experience the lowest levels of danger and highest levels of security. This may lead people to be less vigilant with regard to health practices and may, paradoxically, put people in the safest zones at greater risk of future outbreak. The specific ways in which historical rates of infection influence health 
practices should be explored in future studies that aim to test causal models of COVID-19 health behavior outcomes.

A limitation of the present study is that we selected a relatively arbitrary cutoff to define the three infection density rate zones. Future studies may wish to define zones based on standard deviation units to define upper- and lower- cutoff boundaries. The approach we used here did provide initial evidence that sampling from geographic regions defined by county-level parameters produces meaningful regional differences in the expected direction. As such, there is significant promise in merging online data collection with extant county-level datasets. These two methodologies have up until now remained largely independent of each other. We suggest that there is significant research potential in merging these disparate methodologies. 


\section{References}

Abelman, R. (1996). Standpoint: Can we generalize from generation X? Not! Journal of Broadcasting \& Electronic Media, 40(3), 441-446. doi:10.1080/08838159609364365

Ashton, R. H., \& Kramer, S. S. (1980). Students as surrogates in behavioral accounting research: Some evidence. Journal of Accounting Research, 18(1), 1-15. doi:10.2307/2490389

Ballew, M. T., Goldberg, M. H., Rosenthal, S. A., Gustafson, A., \& Leiserowitz, A. (2019). Systems thinking as a pathway to global warming beliefs and attitudes through an ecological worldview. Proceedings of the National Academy of Sciences, 116(17), 82148219.

Beaver, K. M., \& Wright, J. P. (2011). The association between county-level IQ and county-level crime rates. Intelligence, 39(1), 22-26.

Buhrmester, M. D., Talaifar, S., \& Gosling, S. D. (2018). An evaluation of Amazon's Mechanical Turk, its rapid rise, and its effective use. Perspectives on Psychological Science, 13,149 - 154. https://doi.org/10.1177/1745691617706516

Buhrmester, M., Kwang, T., \& Gosling, S. D. (2011). Amazon's Mechanical Turk: A new source of inexpensive, yet high-quality, data? Perspectives on Psychological Science, 6(1), 3-5.

Chandler, J., Rosenzweig, C., Moss, A.J. et al. Online panels in social science research: Expanding sampling methods beyond Mechanical Turk. Behav Res 51, 2022-2038 (2019).

Davidai, S. (2018). Why do Americans believe in economic mobility? Economic inequality, external attributions of wealth and poverty, and the belief in economic mobility. Journal of Experimental Social Psychology, 79, 138-148. https://doi.org/10.1016/j.jesp.2018.07.012 
Eichstaedt, J. C., Schwartz, H. A., Kern, M. L., Park, G., Labarthe, D. R., Merchant, R. M., ... \& Weeg, C. (2015). Psychological language on Twitter predicts county-level heart disease mortality. Psychological science, 26(2), 159-169.

Frankovic, K. (2020). A majority of Republicans and Democrats are now wearing face masks. YouGov, Retrieved from: https://today.yougov.com/topics/politics/articlesreports/2020/04/23/republicans-anddemocrats-wear-masks

Goldsberry, L., (2017) Understanding designated market area (DMA) and how it impacts your company. https://www.axiapr.com/blog/understanding-designated-market-area-and-howitaffects-your-business Axia.com/blog.

Gosling, S. D., Vazire, S., Srivastava, S., \& John, O. P. (2004). Should we trust web-based studies? A comparative analysis of six preconceptions about Internet questionnaires. American Psychologist, 59(2), 93-104. doi:10.1037/0003-066X.59.2.93

Henrich, J., Heine, S. J., \& Norenzayan, A. (2010). The weirdest people in the world? Behavioral and brain sciences, 33(2-3), 61-83. https://doi.org/10.3758/s13428-019-01273-7

Hu, Z., \& Rao, K. R. (2009). Particulate air pollution and chronic ischemic heart disease in the eastern United States: a county level ecological study using satellite aerosol data. Environmental Health, 8(1), 26.

Jia, H., Muennig, P., \& Borawski, E. (2004). Comparison of small-area analysis techniques for estimating county-level outcomes. American journal of preventive medicine, 26(5), 453460. 
Job, V., Sieber, V., Rothermund, K., \& Nikitin, J. (2018). Age differences in implicit theories about willpower: Why older people endorse a nonlimited theory. Psychology and aging, $33(6), 940$.

Killeen, B. D., Wu, J. Y., Shah, K., Zapaishchykova, A., Nikutta, P., Tamhane, A., ... \& Unberath, M. (2020). A County-level Dataset for Informing the United States' Response to COVID-19. arXiv preprint arXiv:2004.00756.

Kim, D., Subramanian, S. V., Gortmaker, S. L., \& Kawachi, I. (2006). US state-and county-level social capital in relation to obesity and physical inactivity: a multilevel, multivariable analysis. Social science \& medicine, 63(4), 1045-1059.

Lawless, N. M., \& Lucas, R. E. (2011). Predictors of regional well-being: A county level analysis. Social Indicators Research, 101(3), 341-357.

Litman, L., \& Robinson, J. (2020). Conducting Online Research on Amazon Mechanical Turk and Beyond (Vol. 1). SAGE Publications, Incorporated.

Moss, A. J., Rosenzweig, C., Robinson, J., \& Litman, L. (2020). Demographic Stability on Mechanical Turk Despite COVID-19. Trends in Cognitive Sciences.

Muramatsu, N. (2003). County-level income inequality and depression among older Americans. Health services research, 38(6p2), 1863-1884.

Nosek, B. A., Banaji, M. R., \& Greenwald, A. G. (2002). Harvesting implicit group attitudes and beliefs from a demonstration web site. Group Dynamics: Theory, Research, and Practice, 6(1), 101-115. doi:10.1037/1089-2699.6.1.101

Oakes, W. (1972). External validity and the use of real people as subjects. American Psychologist, 27(10), 959-962. doi:10.1037/h0033454 
Plaut, V. C., Markus, H. R., Treadway, J. R., \& Fu, A. S. (2012). The cultural construction of self and well-being: A tale of two cities. Personality and Social Psychology Bulletin, 38(12), 1644-1658.

Pridemore, W. A. (2005). A cautionary note on using county-level crime and homicide data. Homicide Studies, 9(3), 256-268.

Robinson, J., Rosenzweig, C., Moss, A. J., \& Litman, L. (2019). Tapped out or barely tapped? Recommendations for how to harness the vast and largely unused potential of the Mechanical Turk participant pool. PloS one, 14(12).

Sears, D. O. (1986). College sophomores in the laboratory: Influences of a narrow data base on social psychology's view of human nature. Journal of Personality and Social Psychology, 51(3), 515-530. doi:10.1037/0022-3514.51.3.515

Smith, M. A., \& Leigh, B. (1997). Virtual subjects: Using the Internet as an alternative source of subjects and research environment. Behavior Research Methods, 29(4), 496-505. 
Table 1

Sample percentages of face mask wearing, regional differences, party affiliation, and political views

\begin{tabular}{|c|c|c|c|c|}
\hline & \multicolumn{4}{|c|}{ COVID-19 Density } \\
\hline & Whole sample & High & Middle & Low \\
\hline \multicolumn{5}{|l|}{ Wearing face mask } \\
\hline Yes & 79 & 84.9 & 81.1 & 71 \\
\hline \multicolumn{5}{|l|}{ Political party } \\
\hline Democrat & 34.8 & 39.7 & 30.5 & 24.7 \\
\hline Republican & 31.5 & 29.2 & 35.6 & 39.3 \\
\hline Independent & 25.7 & 25.6 & 27.4 & 23.8 \\
\hline Other & 1.6 & 0.8 & 1.4 & 2.5 \\
\hline No preference & 6.5 & 4.7 & 5.1 & 9.7 \\
\hline \multicolumn{5}{|l|}{ Political views } \\
\hline Extremely liberal & 7.2 & 11.4 & 5.9 & 4.4 \\
\hline Liberal & 13.6 & 18 & 12.1 & 10.8 \\
\hline Slightly liberal & 8.4 & 8.9 & 9 & 7.5 \\
\hline Moderate & 25.1 & 24.9 & 26 & 24.4 \\
\hline Slightly conservative & 9.8 & 9.7 & 9.6 & 9.7 \\
\hline Conservative & 16 & 13 & 17.2 & 17.5 \\
\hline Extremely conservative & 11.3 & 7.2 & 13.6 & 13 \\
\hline $\begin{array}{l}\text { I haven't thought about } \\
\text { this }\end{array}$ & 8.7 & 6.9 & 6.5 & 12.7 \\
\hline \multicolumn{5}{|l|}{ Region } \\
\hline $\begin{array}{l}\text { In open country but not } \\
\text { on a farm }\end{array}$ & 13.8 & 5.3 & 9.9 & 26.6 \\
\hline On a farm & 3 & 1.1 & 2.3 & 5.8 \\
\hline $\begin{array}{l}\text { In a small city or town } \\
\text { (fewer than } 50,000 \\
\text { residents) }\end{array}$ & 30.9 & 18 & 27.1 & 47.1 \\
\hline $\begin{array}{l}\text { In a medium size city } \\
\text { ( } 50,000 \text { to } 250,000 \\
\text { residents) }\end{array}$ & 17.4 & 16.1 & 22.3 & 13.6 \\
\hline $\begin{array}{l}\text { In a suburb near a large } \\
\text { village }\end{array}$ & 23.5 & 38.2 & 28.2 & 4.7 \\
\hline $\begin{array}{l}\text { In a large city (more than } \\
250,000 \text { residents) }\end{array}$ & 11.3 & 21.3 & 10.2 & 2.2 \\
\hline
\end{tabular}


Table 2

Basic Sample Demographics

\begin{tabular}{|c|c|c|c|c|}
\hline \multirow[b]{2}{*}{ Age } & \multirow[b]{2}{*}{ Whole sample } & \multicolumn{3}{|c|}{ COVID-19 Density } \\
\hline & & High & Middle & Low \\
\hline $18-29$ & $53.0(17.2)$ & $52.3(17.1)$ & $53.6(17.9)$ & $52.9(16.9)$ \\
\hline \multicolumn{5}{|l|}{ Gender } \\
\hline Male & 45.8 & 48.6 & 48.9 & 39.2 \\
\hline \multicolumn{5}{|l|}{ Race } \\
\hline White & 86.6 & 81.7 & 87.9 & 91.1 \\
\hline Black & 5.3 & 7.5 & 5.6 & 2.2 \\
\hline $\begin{array}{l}\text { American Indian or Alaska } \\
\text { Native }\end{array}$ & 1.5 & 1.1 & 0.8 & 2.5 \\
\hline Asian & 3.5 & 5.5 & 3.1 & 1.9 \\
\hline $\begin{array}{l}\text { Native Hawaiian or Pacific } \\
\text { Islander }\end{array}$ & 0.2 & 0 & 0.3 & 0.3 \\
\hline Other & 2.8 & 4.2 & 2.3 & 1.9 \\
\hline \multicolumn{5}{|l|}{ Hispanic } \\
\hline Yes & 6 & 7.5 & 7.1 & 3.3 \\
\hline \multicolumn{5}{|l|}{ Highest Degree } \\
\hline No college degree & 46.1 & 39.2 & 46 & 52.6 \\
\hline Associate degree & 13.9 & 10.6 & 16.1 & 15 \\
\hline Bachelor's degree & 23.7 & 26.1 & 24.9 & 20.5 \\
\hline Graduate degree & 16.3 & 24.2 & 13 & 11.9 \\
\hline \multicolumn{5}{|l|}{ Annual household income } \\
\hline$<\$ 10 \mathrm{k}$ & 6.31 & 5.3 & 5.9 & 9.2 \\
\hline \$10k-\$19,999 & 6.6 & 7.5 & 9.9 & 11.4 \\
\hline \$20k-\$29,999 & 11.67 & 5.5 & 11.9 & 15 \\
\hline$\$ 30 k-\$ 39,999$ & 10.82 & 10.2 & 11 & 13.3 \\
\hline$\$ 40 \mathrm{k}-\$ 49,999$ & 11.02 & 8.3 & 9.9 & 10 \\
\hline$\$ 50 k-\$ 59,999$ & 11.22 & 7.8 & 8.5 & 9.7 \\
\hline \$60k-\$69,999 & 7.94 & 8.9 & 8.2 & 8.1 \\
\hline$\$ 70 k-\$ 79,999$ & 7.3 & 8 & 8.5 & 7.5 \\
\hline \$80k-\$89,999 & 5.06 & 6.1 & 4.2 & 4.4 \\
\hline \$90k-\$99,999 & 5.16 & 4.4 & 5.1 & 2.8 \\
\hline \$100k-\$149k & 11.97 & 19.9 & 9.9 & 4.4 \\
\hline$>\$ 150 \mathrm{k}$ & 4.92 & 8.9 & 7.1 & 4.2 \\
\hline
\end{tabular}


Table 3

Regression models

Model Set 1: DV = Wearing face mask, each variable as a separate predictor

COVID-19 density

Political party (Rep. vs. Dem.)

Income

Education (College degree vs. no degree)

Age

Political affiliation

County population

\section{OR}

1.47

1.57

1.05

0.67

1.01

0.89

1.24
SE

0.18

0.3

0.023

0.13

0.004

0.03

0.1
95\% CI

1.16-1.87

$1.07-2.30$

1.01-1.10

0.46-0.97

1.00-1.02

0.83-0.96

1.06-1.47

$\quad \mathbf{p}$
$<.05$
$<.05$
$<.05$
$<.05$
$<.05$
$<.05$
$<.05$

Model Set 2: DV = Wearing face mask, all non-county-level predictors entered together

$\begin{array}{lcccc} & \text { OR } & \text { SE } & \mathbf{9 5 \%} \text { CI } & \text { p } \\ \text { Political party (Rep. vs. Dem.) } & 1.51 & 0.31 & 1.01-2.26 & <.05 \\ \text { Income } & 1.04 & 0.026 & 0.98-1.09 & >.05 \\ \text { Education (College degree vs. } & 0.73 & 0.15 & 0.48-1.1 & >.05 \\ \text { no degree) } & 1.01 & 0.005 & 1.00-1.02 & <.05 \\ \text { Age } & & & \end{array}$

Model Set 3: DV = Wearing face mask. County-level predictors added to predictors in Model Set 2

\begin{tabular}{lcccc} 
& $\mathbf{B}$ & $\boldsymbol{S E}$ & $\mathbf{9 5 \%} \mathbf{C I}$ & $\boldsymbol{p}$ \\
COVID-19 density & 1.33 & 0.18 & $1.02-1.74$ & $<.05$ \\
Political party (Rep. vs. Dem.) & 1.4 & 0.29 & $0.93-2.11$ & $>.05$ \\
Income & 1.02 & 0.027 & $0.97-1.07$ & $>.05$ \\
Education (College degree vs. & 0.75 & 0.16 & $0.49-1.08$ & $>.05$ \\
no degree) & 1.01 & 0.004 & $1.00-1.02$ & $<.05$ \\
Age & 1.14 & 0.1 & $0.97-1.36$ & $<.05$ \\
County population & & & \\
\hline
\end{tabular}

Note: Left panel. Predictors regressed onto the binary outcome measure of face mask wearing.

Predictors included age, education, gender, income, race, ethnicity, political party affiliation and political orientation. Each predictor was run separately with no other covariates in the model. 
Only significant predictors are reported in the Level-1 table. Right panel: Level 2 regression in which all predictors were entered as covariates. Note that political affiliation was not included in the level-2 model due to possible multicollinearity with political party. 


\section{Figure 1}

Percent of Participants Living in COVID-19 Infection Density Zones

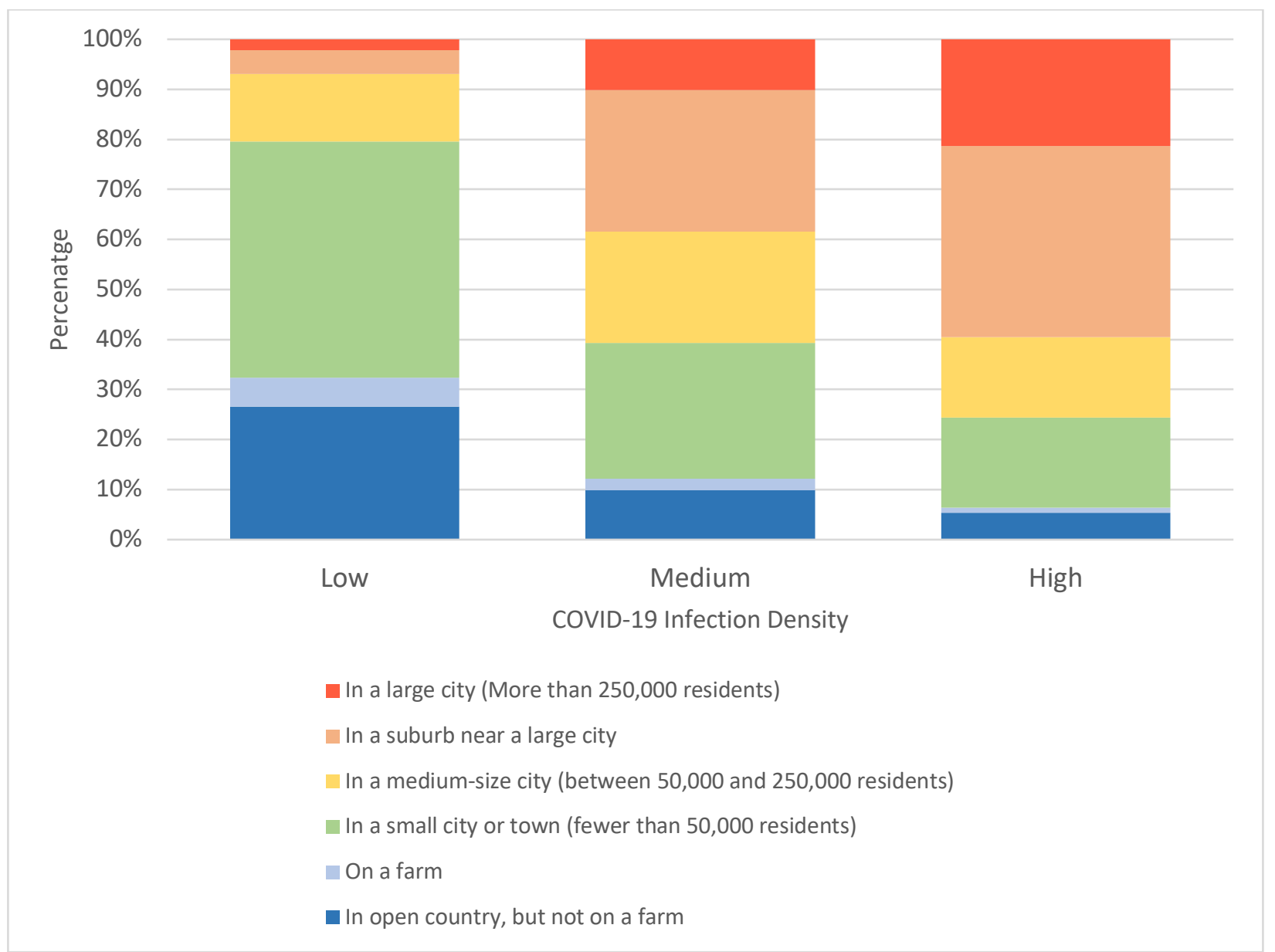




\section{Figure 2}

County-level COVID-19 Density Within Area of Residence

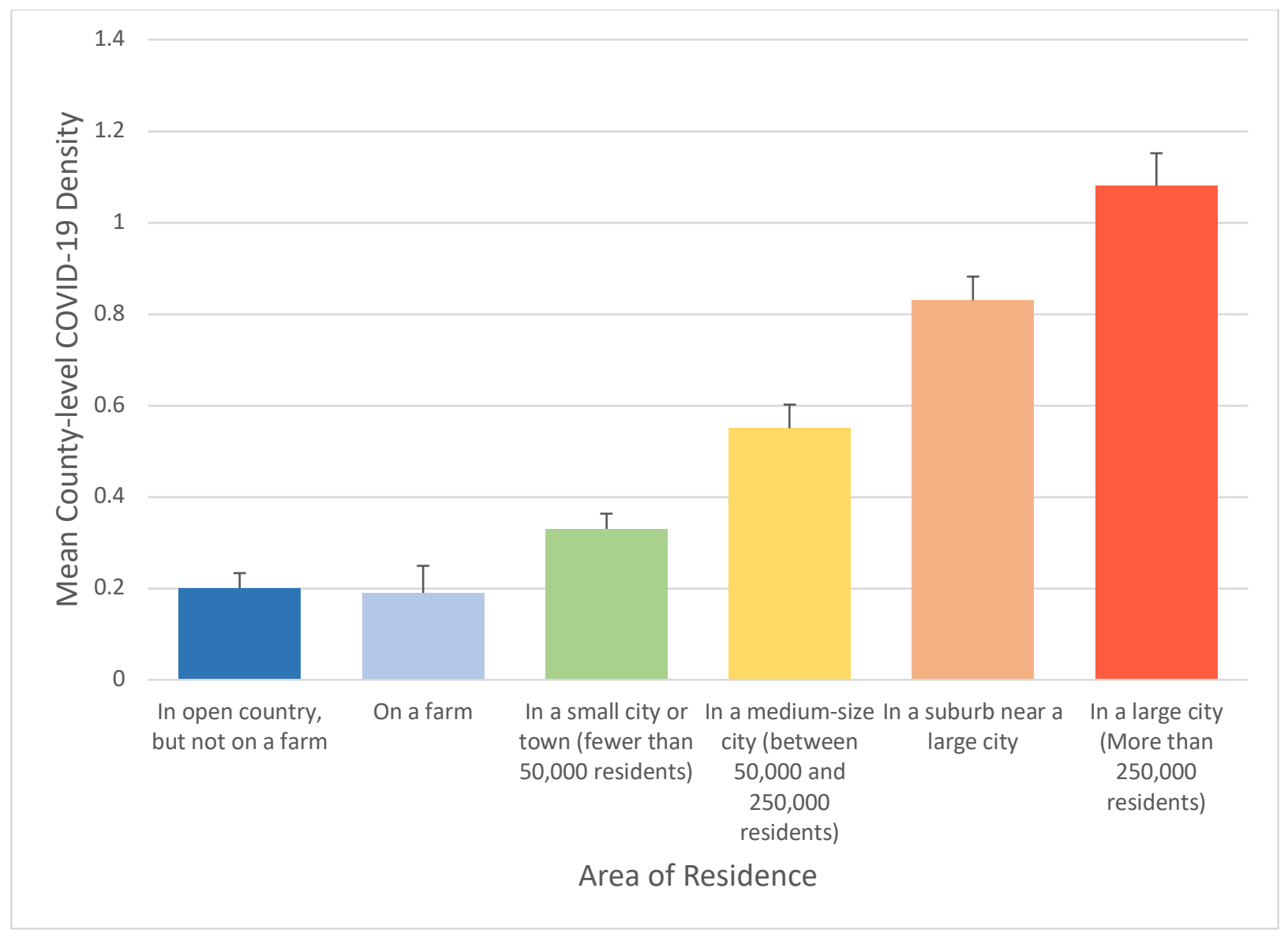

Note: Error bars represent 95\% CIs 